\title{
Research on Brain Control Technology for Wheelchair
}

\author{
Dorian ROTIER ${ }^{1}$,Xiaodong Zhang ${ }^{1, \mathrm{a}}$, Qian $\mathrm{Guo}^{2}$,Liang Yuan $^{3}$ \\ ${ }^{1}$ School of Mechanical Engineering,Xi'an Jiaotong University,710049 Xi'an,Shaanxi,China \\ ${ }^{2}$ School of Mechanical and Electrical Engineering,Xi'an Polytechnic University,710048Xi'an,Shaanxi,China \\ ${ }^{3}$ School of Mechanical Engineering,Xinjiang University,830047 Urumqi,Xinjiang,China
}

\begin{abstract}
This paper proposes a portable brain-computer interface paradigm and builds a portable braincomputer interface device suitable for brain-controlled wheelchairs.According to the characteristics of Emotiv EPOC equipment and its collected EEG,the hardware system of brain-controlled wheelchair was developed,and a real-time detection system of brain-controlled technology was built,which is used to collect EEG signals in real time to realize the wheelchair's motion control.Experimental results show that the accuracy of EEG control can meet the engineering application requirements.
\end{abstract}

\section{Introduction}

Brain Computer Interface (BCI),also known as direct neural Interface or brain-machine interface ${ }^{[1]}$, is an important research field of biomedical engineering in recent years.By extracting specific bioelectrical signals from the brain and decoding them effectively according to the existing theories of cognitive neuroscience, it establishes a direct connection channel between human or animal brains(or the culture of brain cells)and the external instruments or devices to control their movements ${ }^{[2]}$.

It has been a dream of human beings since ancient times to communicate with the outside world directly with the signals of mind activity and realize the control of the surrounding environment.Since the first recording of the EEG by Hans Berger in $1929^{[3]}$,it has been speculated that it might be used for communication and control, allowing the brain to act directly on the outside world without the usual medium - peripheral nerves and limb.However,due to the limitations of the overall technological level at that time and the lack of understanding of the brain's thinking mechanism,little progress has been made in this area.With the improvement of people's understanding of the function of nervous system and the development of computer technology, the research of BCI technology shows an obvious upward trend,especially the two BCI international conferences held in 1999 and 2002,which have pointed out the direction for the development of BCI technology ${ }^{[4]}$.At present,BCI technology has attracted the attention of many scientific and technological workers in the world and is a new research hotspot in biomedical engineering,computer technology and communication ${ }^{[5]}$.

This paper mainly will investigate a human-machine interaction mode with friendly interface,propose a portable brain-machine interface paradigm, and build a portable brain-machine interface device suitable for brain-controlled wheelchairs.Secondly,the hardware development of the brain-controlled wheelchair will be carried out,the real-time detection system of the braincontrolled technology is set up,the adaptive coordinated control system of the motor is constructed,and the corresponding control strategy is also studied.Finally, the experiment for testing E-motiv EPOC and brain electrical signal characteristics,the experiment for brain control wheelchair is done so as to obtain good control ability,respectively.

\section{Schematic design on brain control wheelchair}

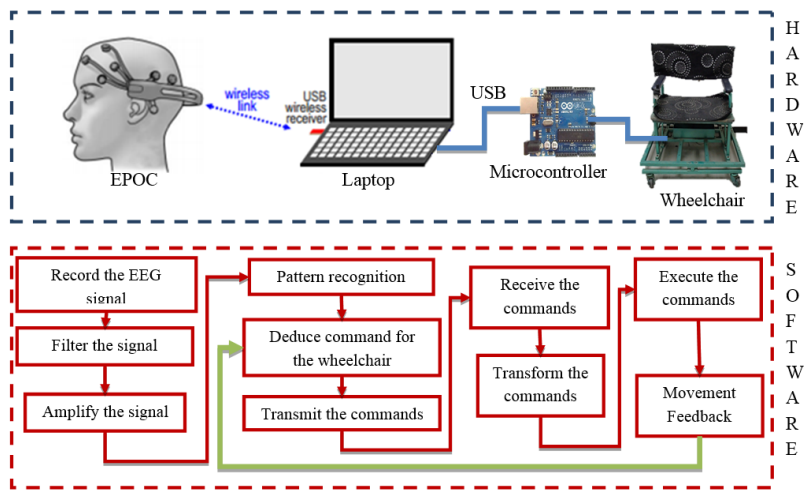

Figure1.Schematic diagram of brain control wheelchair

The schematic diagram of brain control wheelchair(BCW)is presented in Figure1, which has three main parts:the acquisition part of the user brain waves, the related software part for these waves to be processed and analysed,the hardware part for orders to be decided and transmitted to realize concretely the displacements after the analysis of the brain waves. 


\section{Hardware design on brain control wheelchair}

\subsection{EEG recorder}

As you know,the EEG signal is composed of Beta wave,Gamma wave,Alpha wave, Theta wave and Delta wave $^{[6]}$.In general, electroencephalogram (EEG) recording of radio signals is performed by EEG medical grade headset as shown in Figure2(a).For brain control wheelchair,a wireless portable EPOC headset of Emotiv company is presented to collect EEG signals as shown in Figure2(b).Emotiv's EPOC is a wireless portable EEG device with 14 channels, high spatial resolution,dense record array,high quality,raw EEG data,designed in line with actual needs, and is the most credible and costeffective wireless mobile EEG device on the market at present.It measures electrical activity in the brain through electrodes placed on the scalp,collecting electrical potential from the activity of nerve cells, these low potentials, which are then amplified and converted to graphics.

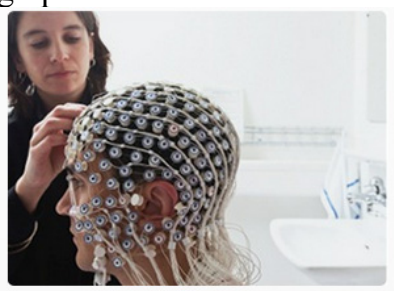

(a)EEG medical grade headset

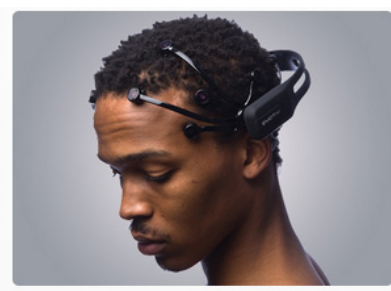

(b)EPOC headset
Figure2.EEG recorder.

\subsection{Processing of EEG data}

To process the raw EEG data provided by the EPOC,a commercial laptop is used to run under Windows8 64 bit with a processor Intel®Core ${ }^{\mathrm{TM}}$ i5-3230M,a memory of 4096 DDR3 RAM (1 600MHz) and a graphic card AMD

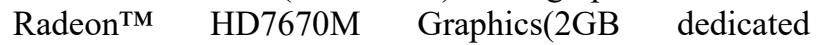
memory).During all the experiment it should be paid attention that all the application running on the computer use less than $50 \%$ of the processor and memory capacity.So it is sure that no slow in the EEG processing will occur.

\subsection{Arduino UNO}

For this application,Arduino UNO is chosen as the microcontroller as shown in Figure3. The microcontroller will act as an interface between the computer communicating by USB and the motor controller of the wheelchair.Arduino UNO is simple,easy to use and economical.At $16 \mathrm{MHz}$ clock speed, it is much faster than the system needs and meets the use requirements. And its sticker price is even less than $\$ 8^{[7]}$.

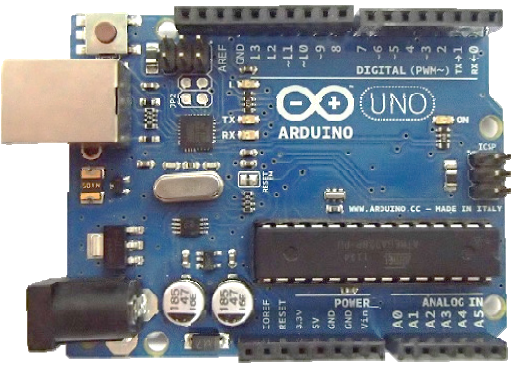

Figure3.Arduino UNO board

\subsection{Actuator}

To execute the command,two brushless motors are used.They are controlled by two motor controllers ZD6716V3,so as to control the speed and the rotation direction of the motors as shown in Figure4.In each motor,there is a Hall sensor, providing a speed signal feedback from the motors,to know concretely the speed of each one.

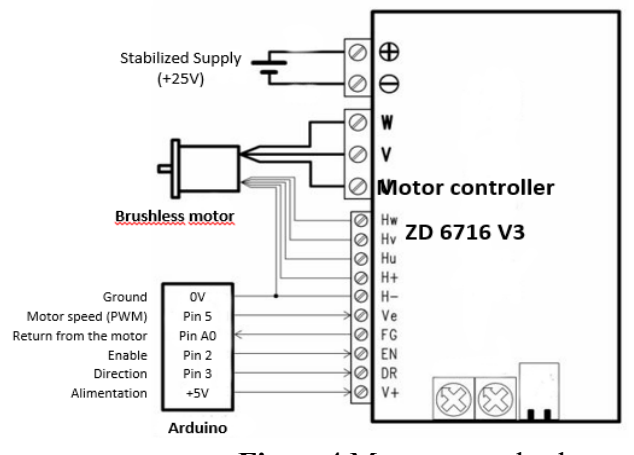

Figure4.Motor control scheme.

\subsection{Design result}

Once all the components are assembled,the hardware design result is shown in Figure5.

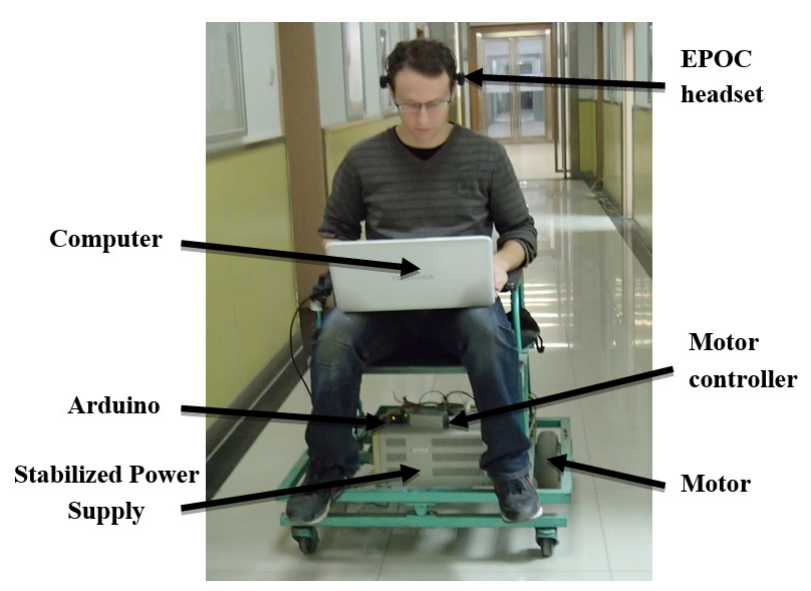

Figure5. The brain control wheelchair prototype.

\section{Software design on brain control wheelchair}




\subsection{Schedule design of software part}

The user's EEG is recorded by the EEG EPOC headset,and the original signal is filtered and amplified by the proprietary software,which provided by the EEG EPOC headset.Then the special software carries on the pattern recognition to the EEG signal,detects the user's movement thought.Once the EEG is decrypted,the results will be sent through the API to the personal software.The software was developed to collect corresponding instructions according to the decrypted EEG.The command is then sent to the wheelchair via Arduino.The brain control software flow chart as shown in Figure6.

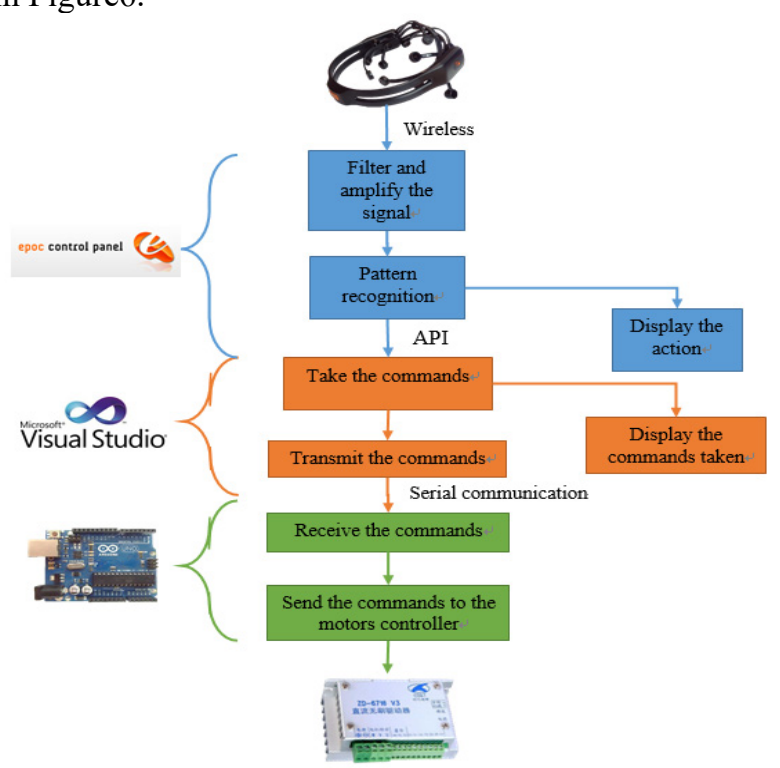

Figure6.The brain control software flow chart

\subsection{Processing of raw EEG input}

The data collected by the EPOC are transferred by an encrypted channel.After a proprietary software call EmoEngine is provided with the EPOC to process and decrypt the raw EEG data.The EmoEngine also provides the battery level of the EPOC,the contact quality of each electrode, the quality of the wireless signal,the gyro data on two axis.For researcher version of EmoEngine,it's also possible to visualize the raw EEG data and record them.Emotiv also provide some software to visualize and use the data such as the Control Panel to visualize the processed data,EmoKey to link the processed EEG data with a key stroke.

But the more important for the project is the $\mathrm{C}++$ Application Programming Interface (API) provided by EmoEngine to develop personal application,as shown in Figure7.The EmoEngine decrypt the EEG data and make the processed data accessible for every application running on the computer through the API.To help the development of new application,Emotiv provides examples using $\mathrm{C}++$,Java,NET, and Mat Lab language.

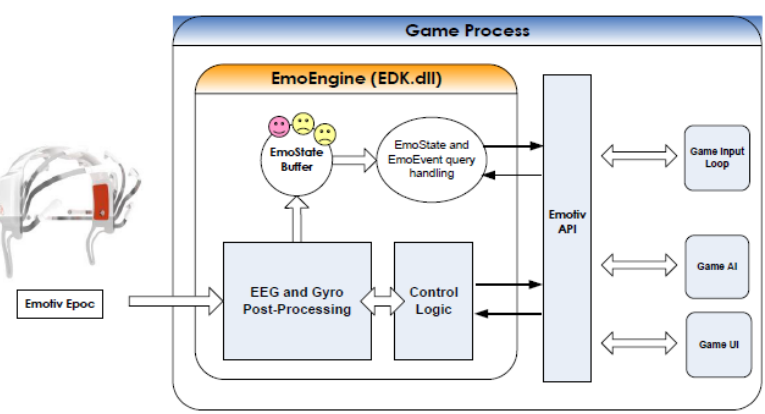

Figure7.Diagram of Emotiv API used for a videogame

\subsection{Command}

Once the EEG signal has been decrypted by the EmoEngine,the decrypted data are available through the EmoEngine Application Programming Interface(API).An informatics program is designed using the EmoEngine data through the API to issue commands for the wheelchair.The interest of such program is to expand dramatically the capacity of the EPOC,there is no more constraint induced by a proprietary program,everything is possible, it just need to be coded. Every data of the EPOC can be used at the same time and personal algorithm or filter can be developed to issue proper commands. With a personal program the process is also entirely under control and fully understandable.

\subsection{Design results and analysis}

The operation of the software part and the relations between the different programs are resumed as shown in figure8.

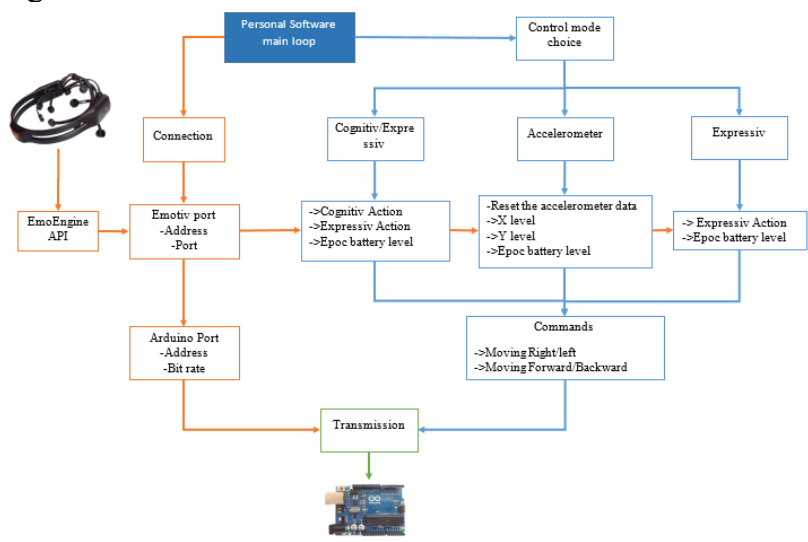

Figure8.Functional diagram of personal software

Using directly the EmoEngine information through the API,the software is really effective and reactive. Between the reception of the user action by EmoEngine and transmitting the command to the motor controller,it takes less than $50 \mathrm{~ms}$.

\section{Experimental Verification}

\subsection{Reliability of the EEG data}


Before controlling the wheelchair, we have to determine which EEG data is the more reliable. The users is comfortably seated on a chair, his feet lay on the floor and his hand on the armrests, as shown in Figure9. He is asked during all the experiment to move as little as possible. The helmet is carefully placed on his head following the instruction of the EPOC manual. This is really critical to have reliable and repeatable results, therefore a special attention is also paid to this step.

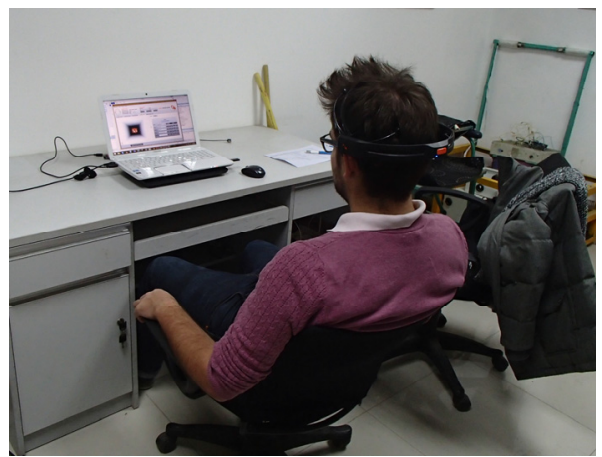

Figure 9. The configuration of the experiment 1

To see if clench, smirk left/right are suitable to control the system, the graphic power versus time are plotted for two experiments $(20 \mathrm{~s})$ and for each action, it is appropriate for user 1 and 2 to grasp the threshold of 0.015 , but for user 3 , the detection ability is good, but it is constant, so the threshold of 0.003 is more appropriate.

\subsection{Test of brain control wheelchair}

To confirm the all design of the $\mathrm{BCW}$, it is put to the test. The three same control modes are tested on the two circuits. The user is seated on the wheelchair with the laptop,wearing the EPOC as shown in Figure10.

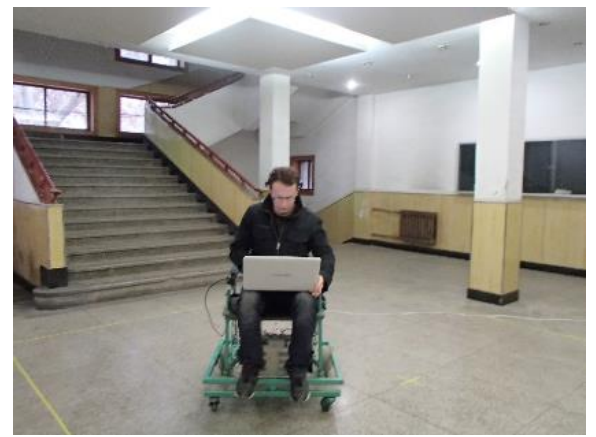

Figure10.The configuration of the experiment 2

Here are the results of the experiment 2, with D the percentage for comparison of the time to finish the circuit with the reference time set using the joystick, as shown in Table1.

The experimental results show the control mode using accelerometer is much easier to use. Note that the number of off-track and shock for all control modes and circuit are close to zero.The design is completely validated by the results of this experiment,the user has a good control of the wheelchair and the speed is satisfactory.For the slowest mode control and for the circuit requesting the more change of direction, it is just $67 \%$ slower than the time of reference established with the joystick.

Table1The results of the experiment 2 for the two circuits.

\begin{tabular}{|c|c|c|c|c|c|c|c|c|c|c|c|}
\hline & \multicolumn{2}{|c|}{ Joystick } & \multicolumn{3}{|c|}{ Accelerometer } & \multicolumn{3}{|c|}{ Expressiv } & \multicolumn{3}{|c|}{ Expressiv/EEG } \\
\hline & & $\begin{array}{l}\text { Shock } \\
\text { Ooff }\end{array}$ & $\begin{array}{l}\mathrm{t} \\
\text { (s) }\end{array}$ & $\begin{array}{l}\text { Shock } \\
\text { Ooff }\end{array}$ & $\mathrm{D}$ & $\begin{array}{l}\mathrm{t} \\
\mathrm{(s}\end{array}$ & $\begin{array}{l}\text { Shock } \\
\text { /Off }\end{array}$ & $\mathrm{D}$ & $\begin{array}{l}\mathrm{t} \\
\mathrm{(s}\end{array}$ & $\begin{array}{l}\text { Shock } \\
\text { /Off }\end{array}$ & $\mathrm{D}$ \\
\hline $\begin{array}{c}\text { Circuit } \\
1\end{array}$ & 37 & 0 & 48 & 0 & $30 \%$ & 52 & 0 & $41 \%$ & 55 & 0 & $49 \%$ \\
\hline $\begin{array}{c}\text { Circuit } \\
2\end{array}$ & 85 & 0 & 100 & 0 & $18 \%$ & 137 & 0 & $61 \%$ & 145 & 1 & $67 \%$ \\
\hline
\end{tabular}

\section{Conclusions}

The results show that the portable brain-computer interface designed in this paper is a simple and effective brain-computer interface device, and the accuracy of EEG control can meet the engineering application requirements.At the same time, the system has a high cost performance and costs about $\$ 500$, which is suitable for industrial development.

\section{Acknowledgement}

The authors are grateful for the support provided by the Assistance Xinjiang Science and Technology Project of Xinjiang province, China (2017E0284).

\section{References}

1. PHELPS, Michael E., MAZZIOTTA, John C., Positron emission tomography: human brain function and biochemistry. Science, 1985, vol. 228, no 4701, p. 799-809.

2. WANG, Yijun, GAO, Xiaorong, HONG, Bo, Braincomputer interfaces based on visual evoked potentials. Engineering in Medicine and Biology Magazine, IEEE, 2008, vol. 27, no 5, p. 64-71.

3. WOLPAW, Jonathan R., Brain-computer interfaces as new brain output pathways. The Journal of Physiology, 2007, vol. 579, no 3, p. 613-619.

4. GALÁN, Ferran, NUTTIN, Marnix, LEW, Eileen, A brain-actuated wheelchair: asynchronous and non-invasive brain-computer interfaces for continuous control of robots. Clinical Neurophysiology, 2008, vol. 119, no 9, p. 21592169.

5. Brice Rebsamen, A Brain Controlled Wheelchair to Navigate in Familiar Environments, HumanComputer Interaction. National university of Singapore, 2009. p.56

6. R. Lievesley, M. Wozencroft, and D. Ewins, The Emotiv EPOC neuroheadset: an inexpensive method of controlling assistive technologies using facial expressions and thoughts, Journal of Assistive Technologies, 2011, vol. 5, no. 2, pp. 6782.

7. TANAKA, Kazuo, MATSUNAGA, Kazuyuki, WANG, Hua O, Electroencephalogram-based 
control of an electric wheelchair. Robotics, IEEE

Transactions on, 2005, vol. 21, no 4, p. 762-766. 